\title{
Training in flexible intensive insulin management improved glycaemic control and quality of life in type 1 diabetes
}

DAFNE Study Group. Training in flexible, intensive insulin management to enable dietary freedom in people with type 1 diabetes: dose adjustment for normal eating (DAFNE) randomised controlled trial. BMJ 2002;325:746-9.

\author{
QUESTION: Does training in flexible intensive insulin management (combining dietary \\ freedom and insulin adjustment) improve glycaemic control and quality of life in patients \\ with type 1 diabetes?
}

\section{Design}

Randomised (allocation concealed), unblinded, controlled trial with follow up at 6 months (Dose Adjustment for Normal Eating [DAFNE] trial).

\section{Setting}

3 hospital diabetes clinics in Sheffield, Northumbria, and London, UK.

\section{Patients}

169 patients $>18$ years of age with clinical features of type 1 diabetes, moderate or poor glycaemic control (glycated haemoglobin $\left[\mathrm{HbA}_{1 \mathrm{c}}\right]$ 7.5-12\%), and duration of diabetes $>2$ years without advanced complications. Exclusion criteria were inability to understand English or identify hypoglycaemia, severe psychiatric illness, and pregnancy. 136 patients $(80 \%)$ completed baseline and 6 month assessments (mean age 40 y, 56\% women).

\section{Intervention}

84 patients were allocated to the intervention, which comprised a 5 day skills course delivered by 2-3 educators (diabetes specialist nurses or dietitians) to groups of 6-8 participants in each centre. Patients were taught to adjust their insulin by matching it to the desired carbohydrate intake at each meal (rather than adjusting the timing and content of meals to match prescribed doses of insulin). 85 patients were allocated to usual care for 6 months, after which they received the training intervention.

\section{Main outcome measures}

$\mathrm{HbA}_{1 c}$ concentrations, patient recorded episodes of severe hypoglycaemia (ie, episodes causing coma or requiring the assistance of another person), and impact of diabetes on quality of life (Audit of DiabetesDependent Quality of Life [ADDQoL] questionnaire, 19 point scale).

\section{Main results}

At 6 months, patients in the intervention group had greater improvement in glycaemic control (mean difference in $\mathrm{HbA}_{1 \mathrm{c}} 1.0 \%, 95 \%$ CI 0.5 to 1.4 ) and weighted impact of diabetes on quality of life (mean difference between groups $0.4, \mathrm{p}<0.01$ ) than the usual care group. The groups did not differ for episodes of severe hypoglycaemia $(18 \% v 15 \%, \mathrm{p}=0.68)$.

\section{Conclusion}

In patients with type 1 diabetes, training in flexible intensive insulin management (combining dietary freedom and insulin adjustment) improved glycaemic control and quality of life at 6 months.

\section{COMMENTARY}

Like the much larger Diabetes Control and Complications Trial (DCCT), ${ }^{1}$ the DAFNE study shows that glycaemic control can be improved by giving people with type 1 diabetes the skills to manage their condition. The DAFNE study also suggests that this improvement can be achieved without a significant increase in severe hypoglycaemia. This finding contrasts with the DCCT, which found a 3 fold increase in the incidence of hypoglycaemia in the intensive insulin group. However, this difference may be the result of differences in how the 2 studies measured hypoglycaemia. Quality of life also improved in the DAFNE trial, again in contrast to the DCCT, which found no change in quality of life.

The strengths of the DAFNE study include its randomised controlled design and $80 \%$ follow up at 6 months. It is also notable that participants were discharged to their usual system of care after attending the 5 day course, indicating that the effects on glycaemic control can be maintained in "normal" life. However, the study is limited by the lack of an economic analysis. Further study is also needed to identify which elements of the "package" were beneficial.

The DAFNE study is important because it provides evidence of an effective education strategy for patients with type 1 diabetes, which has the potential to be implemented in everyday clinical practice. Unfortunately, the study is not relevant to patients with type 2 diabetes because of differences in the management of the diseases. Type 1 diabetes is an insulin deficiency disorder requiring insulin replacement. Type 2 diabetes requires dietary manipulation and lifestyle changes (eg, eating behaviour and activity levels) to achieve good glycaemic control. Promoting dietary freedom in this context is probably not desirable! However, the $1 \%$ reduction in $\mathrm{HbA}_{1 c}$ achieved in the DAFNE trial is desirable for people with both types of diabetes, as the incidence of diabetes complications is likely to be reduced. Further research on the effectiveness of a modified DAFNE course for patients with type 2 diabetes is warranted.

Maggie Watkinson, RGN, MSc Editor, Journal of Diabetes Nursing Diabetes Clinical Nurse Specialist Diabetes Centre Musgrove Park Hospital Taunton, $U K$

1 The effect of intensive treatment of diabetes on the development and progression of long-term complications in insulin-dependent diabetes mellitus. The Diabetes Control and Complications Trial Research Group. $N$ Engl J Med 1993;329:977-86. 